\title{
Modulation of T-cell responses to alloantigens by TR6/DcR3
}

\author{
Jun Zhang, ${ }^{1}$ Theodora W. Salcedo, ${ }^{1}$ Xiaochun Wan, ${ }^{2}$ Stephen Ullrich, ${ }^{1}$ Bugen Hu, ${ }^{1}$ \\ Theresa Gregorio, ${ }^{1}$ Ping Feng, ${ }^{1}$ Shijie Qi, ${ }^{2}$ Huifang Chen, ${ }^{2}$ Yun Hee Cho, ${ }^{1}$ Yuling Li, ${ }^{1}$ \\ Paul A. Moore, ${ }^{1}$ and Jiangping $\mathrm{Wu}^{2,3,4}$
}

\author{
${ }^{1}$ Human Genome Sciences Inc., Rockville, Maryland, USA \\ ${ }^{2}$ Research Center, and \\ ${ }^{3}$ Nephrology Service, Centre Hospitalier de l’Université de Montréal (CHUM), Notre-Dame Hospital, University of Montreal, \\ Montreal, Canada \\ ${ }^{4}$ Department of Surgery, McGill University, Montreal, Canada
}

Address correspondence to: Jun Zhang, Human Genome Sciences, 9410 Key West Avenue, Rockville, Maryland 20850, USA. Phone: (301) 309-8504 ext. 2107; Fax: (301) 294-4843; E-mail: jun_zhang@hgsi.com;

or to: Jiangping Wu, Laboratory of Transplantation Immunology, Pavilion Deseve, Rm. Y5616, Research Center, CHUM, Notre-Dame Hospital, 1560 Sherbrooke Street East, Montreal, Quebec, H2L 4MI Canada.

Phone: (514) 890-8000 ext. 25164; Fax: (514) 412-7596; E-mail: jianping.wu@umontreal.ca.

Jun Zhang, Theodora Salcedo, and Xiaochun Wan contributed equally to this work.

Received for publication January 8, 2001, and accepted in revised form April 30, 2001.

TR6 (DcR3) is a new member of the TNF receptor (TNFR) family that lacks a transmembrane domain
in its sequence, indicating that it is a secreted molecule. TR6 can bind to FasL and prevent FasL-
induced apoptosis; it can also associate with LIGHT, another TNF family member. The role of TR6
in immune responses was investigated in this study. According to flow cytometry, recombinant
human TR6-Fc binds to human LIGHT expressed on 293 cells or on activated human T cells and
competes with the LIGHT receptor TR2 for the binding to LIGHT on these cells. Human TR6 could
cross-react with mouse LIGHT in immunoprecipitation. TR6-Fc also downregulates cytotoxic T lym-
phocyte activity in vitro and graft-versus-host responses in mice. Moreover, TR6-Fc modulates lym-
phokine production by alloantigen-stimulated mouse T cells. TR6-Fc ameliorated rejection response
to mouse heart allograft. These results indicate that TR6 can dampen T-cell responses to alloanti-
gens. Such regulatory effects of TR6 probably occur via interference with interaction between pairs
of related TNF and TNFR family members, LIGHT/TR2 being one of the possible candidate pairs.

J. Clin. Invest. 107:1459-1468 (2001).

\section{Introduction}

TR6 (also called DcR3) is a new member of the TNF receptor (TNFR) family. TR6 lacks an apparent transmembrane domain in its sequence and is likely a secreted protein (1). The mRNA of TR6 is expressed at high levels in several normal human tissues such as the stomach, spinal cord, colon, lymph node, and spleen (1, 2 ), whereas its mRNA expression in the thymus is weak, and in peripheral blood lymphocytes is undetectable. Recombinant TR6 fused with an $\operatorname{IgG}_{1} \mathrm{Fc}_{\mathrm{c}}$ domain can inhibit the interaction between Fas and FasL and prevent FasL-induced apoptosis in lymphocytes and several tumor cell lines (1). The latter suggests that certain tumors may escape FasL-dependent immunocytotoxic attack by overexpressing TR6.

TR6 can also bind to LIGHT, which is a member of the TNF family (3). LIGHT is a type II transmembrane protein, and its protein is expressed on activated $\mathrm{T}$ cells (4) and immature dendritic cells (5). It is a ligand for both TR2/HVEM and LTßR (4). LIGHT was found to induce apoptosis in cells expressing both TR2 and LT $\beta R$, but not in cells expressing only TR 2 or only LT $\beta R$ (6). However, a recent report by Rooney et al. (7) raised doubt about this conclusion by showing that LT $\beta R$ is necessary and sufficient for LIGHT-mediated apoptosis of tumor cells. In any case, as LT $\beta R$ is not expressed on lymphocytes (8), LIGHT has no demonstrated or perceived apoptotic effect on these cells.

Recent studies show that LIGHT can modulate T-cell responses via TR2, which is constitutively expressed at both protein and mRNA levels in most lymphocyte subpopulations including CD4 and CD8 T cells $(9,10)$. Soluble LIGHT enhances a three-way MLR (11). LIGHT expressed on COS cells or anchored on solid phase augments T-cell proliferation and lymphokine production $(5,12)$. Molecules that can presumably interfere with the interaction between LIGHT and TR2 were found to downregulate T-cell responses. For example, an antagonistic $\mathrm{mAb}$ against TR2 represses proliferation and lymphokine production by CD4 T cells (9); soluble recombinant TR2-Fc inhibits a three-way $\operatorname{MLR}(9,10)$ or dendritic cell-stimulated alloresponse of the T cells (5); soluble LT $\beta$ R-Fc inhibits solid-phase LIGHT-augmented T-cell proliferation (12); and in vivo administration of LT $\beta$ R-Fc leads to amelioration of mouse graft-versus-host disease (12). 
In the latter two cases, human $\mathrm{Fc}$ was used in constructing the mouse recombinant LT $\beta \mathrm{R}-\mathrm{Fc}$, and the protein was tested in a mouse model.

Because of the observation that TR6 is able to associate with LIGHT, and that LIGHT can regulate T-cell responses, we asked whether TR6 could modulate certain T-cell functions. Our data show that TR6, in addition to its previous reported role as a decoy receptor in apoptosis, may also play an important role in regulation of immune responses.

\section{Methods}

Mice. Two- to four-month-old female C57BL/6 $\left(\mathrm{H}-2^{\mathrm{b}}\right)$, $\mathrm{BALB} / \mathrm{c}\left(\mathrm{H}-2^{\mathrm{d}}\right)$, and BALB/c $\times$ C57BL/6 F1 (H-2 $\left.{ }^{\mathrm{bxd}}\right)$ mice were purchased from The Jackson Laboratory (Bar Harbor, Maine, USA) or Charles River (LaSalle, Quebec, Canada). 2C TCR transgenic mice were bred in our animal facility as described elsewhere (13).

Expression and purification of the human TR6-Fc fusion protein. Full-length human TR6 cDNA (amino acids 1-300) was PCR amplified using gene-specific primers, fused to the sequence coding for the hinge and $\mathrm{Fc}$ domain of human $\operatorname{IgG}_{1}$ and subcloned into a baculovirus expression vector $\mathrm{pA} 2$. The construct was named pA2-Fc:TR6. Sf9 cells infected pA2-Fc:TR6 were grown in a bioreactor in media $(100 \mathrm{~L})$ containing $1 \%$ ultralow $\operatorname{IgG}$ serum. Conditioned culture supernatant from the bioreactor was harvested by continuous flow centrifugation. The supernatant was adjusted to $\mathrm{pH} 7.0$, and it was filtered through 0.22 $\mu \mathrm{m}$ filters and loaded onto a 30-ml bed-volume protein A column (BioSepra Ceramic HyperD; Life Technologies Inc., Rockville, Maryland, USA) previously conditioned with $20 \mathrm{mM}$ phosphate buffer containing $0.5 \mathrm{M} \mathrm{NaCl}$ ( $\mathrm{pH}$ 7.2). The column was washed with 15 column volumes (CV) of this buffer followed by $5 \mathrm{CV}$ of $0.1 \mathrm{M}$ sodium citrate ( $\mathrm{pH}$ 5.0). TR6-Fc was eluted with $0.1 \mathrm{M}$ citric acid ( $\mathrm{pH} 2.4$ ), and 2-ml fractions were collected into tubes containing $0.6 \mathrm{ml}$ Tris- $\mathrm{HCl}(\mathrm{pH}$ 9.2). The TR6-Fc-positive fractions were determined by SDS-PAGE. The peak fractions were pooled and concentrated with a protein A column (7- $\mathrm{ml}$ bed volume) as described earlier here. The concentrated TR6Fc was loaded onto a 90-ml bed-volume Superdex 200 column (Amersham Pharmacia Biotech, Piscataway, New Jersey, USA) and eluted with PBS containing 0.5 $\mathrm{M} \mathrm{NaCl}$. TR6-Fc-positive fractions were determined by nonreducing SDS-PAGE. The pooled positive fractions were dialyzed against $12.5 \mathrm{mM}$ HEPES buffer (pH 5.75) containing $50 \mathrm{mM} \mathrm{NaCl}$. The dialysate was then passed through a $0.2-\mu \mathrm{m}$ filter (Minisart; Sartorius AG, Goettingen, Germany) followed by a Q15Xanion exchange membrane (Sartobind membrane; Sartorius AG, Goettingen, Germany).

Expression and purification of full-length human TR6 (without $F$ c). The full-length TR6 cDNA was PCR amplified and cloned into the baculovirus expression vector $\mathrm{pA} 2$ as described earlier here. Sf9 cells were infected with the viral construct, and the culture supernatant of the infected cells was loaded onto a Poros HS-50 column equilibrated in a buffer containing $50 \mathrm{mM}$ Tris- $\mathrm{HCl}$ $(\mathrm{pH} 7)$ and $0.1 \mathrm{M} \mathrm{NaCl}$. The column was washed with $0.1 \mathrm{M} \mathrm{NaCl}$ and eluted stepwise with $0.3 \mathrm{M}, 0.5 \mathrm{M}$, and $1.5 \mathrm{M} \mathrm{NaCl}$. The eluted fractions were analyzed by SDSPAGE, and the $0.5 \mathrm{M} \mathrm{NaCl}$ fraction containing TR6 protein was diluted and loaded onto a set of anion (Poros HQ-50; Applied Biosystems, Foster City, California, USA) and cation (Poros CM-20; Applied Biosystems) exchange columns in a tandem mode. TR6 was eluted from the CM-20 column with a linear gradient from $0.2 \mathrm{M}$ to $1.0 \mathrm{M} \mathrm{NaCl}$.

Expression and purification of buman TR2-Fc, MCIF-Fc, and TR11-Fc fusion proteins. The cDNA sequences coding for the extracellular domain of TR2 (amino acids 1-192), the extracellular domain of TR11 (amino acids $1-164$ ) and a $\beta$ chemokine MCIF (also called HCC-1; amino acids 1-92) were fused with the cDNA sequence coding for the $\mathrm{Fc}$ domain of human $\mathrm{IgG}_{1}$ and then cloned into a eukaryotic expression vector $\mathrm{pC} 4$. The construct was stably transfected into $\mathrm{CHO}$ cells. The $\mathrm{Fc}$ fusion proteins from the $\mathrm{CHO}$ supernatant were purified with methods used for TR6-Fc.

Expression and purification of the human LIGHT protein. The coding sequence of the natural secreted form of LIGHT (amino acids 83-240) was cloned into a prokaryotic expression vector, $\mathrm{pHE} 4$, and expressed in Escherichia coli. Inclusion bodies from the transformed bacteria were dissolved for $48-72$ hours at $4{ }^{\circ} \mathrm{C}$ in 3.5 $\mathrm{M}$ guanidine hydrochloride containing $100 \mathrm{mM}$ Tris$\mathrm{HCl}(\mathrm{pH} 7.4)$, and $2 \mathrm{mM} \mathrm{CaCl}_{2}$. The solution was quickly diluted with $20-30$ volumes of a buffer containing $50 \mathrm{mM}$ Tris- $\mathrm{HCl}(\mathrm{pH} 8.0)$ and $150 \mathrm{mM} \mathrm{NaCl}$ (adjusted to $\mathrm{pH}$ 6.6). and chromatographed with a Poros HS-50 column. The protein was eluted with 3-5 $\mathrm{CV}$ of a stepwise gradient of $300 \mathrm{mM}, 700 \mathrm{mM}$, and $1,500 \mathrm{mM} \mathrm{NaCl}$ in $50 \mathrm{mM}$ MES at $\mathrm{pH}$ 6.6. The fraction eluted with $0.7 \mathrm{M} \mathrm{NaCl}$ was diluted threefold with water and applied to a set of Poros HQ-50 and Poros CM-20 ion exchange columns in a tandem mode. The Poros CM-20 column was eluted with 10-20 CV of a linear gradient from $50 \mathrm{mM}$ MES ( $\mathrm{pH}$ 6.6), $150 \mathrm{mM}$ $\mathrm{NaCl}$ to $50 \mathrm{mM}$ Tris- $\mathrm{HCl}(\mathrm{pH}$ 8), and $500 \mathrm{mM} \mathrm{NaCl}$. Fractions containing purified LIGHT as analyzed by SDS-PAGE were combined.

Quality control of the recombinant proteins. The endotoxin levels in the purified recombinant proteins were determined by the LAL assay on an LAL-5000 Automatic Endotoxin Detection System (Associates of Cape Cod, Falmouth, Massachusetts, USA), according to a standard procedure recommended by the manufacturer. All the recombinant proteins were subjected to $\mathrm{NH}_{2}-$ terminal sequencing using an ABI-494 sequencer (Applied Biosystems) to confirm their authenticity. The proteins was dialyzed against PBS containing 20\% (vol/vol) glycerol for storage at $-80^{\circ} \mathrm{C}$. For applications such as CTL, cytokine secretion, and heart transplantation, the proteins were subsequently dialyzed against PBS to remove the glycerol in the solution. 
BIAcore analysis. The binding of human LIGHT to human TR6, TR6-Fc, or TR2-Fc was assessed by BIAcore analysis (BIAcore Inc., Piscataway, New Jersey, USA). TR6, TR6-Fc, or TR2-Fc was covalently conjugated to BIAcore sensor flow cells (CM5 chip) via amine groups using $N$-ethyl- $N$-(dimethylaminopropyl) carbodiimide/ $N$-hydroxysuccinimide. Various dilutions of LIGHT were passed through the TR6-, TR6Fc-, or TR2-Fc-conjugated flow cells at $15 \mu \mathrm{l} / \mathrm{min}$ for a total volume of $50 \mu \mathrm{l}$. The amount of bound protein was determined during washing of the flow cell with HBS buffer (10 mM HEPES [pH 7.4], $150 \mathrm{mM} \mathrm{NaCl}$, 3.4 mM EDTA, 0.005\% Surfactant P20). The flow-cell surface was regenerated by washing off the bound proteins with $20 \mu \mathrm{l}$ of $10 \mathrm{mM}$ glycine- $\mathrm{HCl}$ at $\mathrm{pH}$ 2.3. For kinetic analysis, the flow cells were tested at different flow rates and with different densities of the conjugated TR6-Fc or TR2-Fc proteins. The association, dissociation, and equilibrium constants were determined according a kinetic evaluation program in the BiaEvaluation 3 software (BIAcore Inc.) using a 1:1 binding model and the global analysis method.

Generation of stable cell lines that express buman LIGHT. The full-length human LIGHT gene was PCR amplified and subcloned into pcDNA3.1. The parental vector and the LIGHT expression vectors were then transfected into 293F cells (Life Technologies Inc., Grand Island, New York, USA) using Lipofectamine (Life Technologies Inc.), and stable clones resistant to $0.5 \mathrm{mg} / \mathrm{ml}$ geneticin were selected.

Flow cytometry. Cells $\left(1 \times 10^{6}\right)$ were incubated with 50 ng Fc-fusion proteins in $100 \mu \mathrm{l}$ FACS buffer (d-PBS with $0.1 \%$ sodium azide and $0.1 \%$ BSA) for $15-20 \mathrm{~min}$ utes at room temperature. The cells were washed once and reacted with goat $\mathrm{F}(\mathrm{ab})_{2}$ anti-human IgG (Southern Biotechnology, Birmingham, Alabama, USA) for 15 minutes at room temperature. After washing, the cells were resuspended in $0.5 \mu \mathrm{g} / \mathrm{ml}$ propidium iodide, and live cells were gated and analyzed on a FACScan (BD Biosciences, Mansfield, Massachusetts, USA).

Stimulation of buman T cells for LIGHT expression. Briefly, $T$ cells were purified from human peripheral blood and stimulated with anti-CD3 in the presence of rhuIL-2 for 5 days. The cells were restimulated with PMA (1 $\mu \mathrm{g} / \mathrm{ml})$ and ionomycin $(1 \mu \mathrm{g} / \mathrm{ml})$ for an additional 4 hours. LIGHT expression on the cells was assessed by the binding of TR6-Fc, TR2-Fc, or TR11-Fc to the cells using flow cytometry.

Immunoprecipitation. The extracellular regions of human LIGHT (amino acids 83-240), mouse LIGHT (amino acids 81-239, GenBank accession number AB029155), and human BLyS (amino acids 134-285; ref. 14) were PCR-amplified and fused at their $\mathrm{NH}_{2}$-terminus to an isoleucine zipper sequence known to enhance the biologic activity of soluble CD40L (15). The $\mathrm{NH}_{2}$-terminus of the isoleucine zipper was then fused with the FLAG epitope preceded by a heterologous signal peptide. The constructs of these FLAGisoleucine zipper-LIGHT/BLyS fusion proteins were created in a mammalian expression vector and were transfected into HEK 293T cells using Lipofectamine Plus. Conditioned supernatants ( $400 \mu \mathrm{l} /$ sample) were collected and subjected to immunoprecipitation with $2 \mu \mathrm{g}$ of purified recombinant TR6-Fc or B cell maturation antigen-Fc (BCMA-Fc) proteins (16). Immune complexes were retrieved by Protein A/G agarose (Santa Cruz Biotechnology, Santa Cruz, California, USA) and subjected to Western Blotting with an anti-FLAG mAb (Clone M2; Sigma Chemical Co., St. Louis, Missouri, USA). The signals were visualized by enhanced chemiluminescence (Amersham Pharmacia Biotech).

Murine graft-versus-host reaction. The F1 of C57BL/ $6 \times \mathrm{BALB} / \mathrm{c}$ mice $\left(\mathrm{H}-2^{\mathrm{bxd}}\right)$ were transfused intravenously with $1.5 \times 10^{8}$ spleen cells from $\mathrm{C} 57 \mathrm{BL} / 6$ mice $\left(\mathrm{H}-2^{\mathrm{b}}\right)$ on day 1. TR6-Fc or a control fusion protein was administered intravenously daily for 9 days at 3 $\mathrm{mg} / \mathrm{kg} / \mathrm{d}$ starting 1 day before the transfusion. The spleens of the recipient F1 mice were harvested on day 9 for measuring the weight and splenocytes were prepared for in vitro proliferation and cytokine assays.

Ex vivo mouse splenocyte proliferation. Single splenocyte suspensions from normal and the transfused F1 mice were cultured in triplicate in 96-well flat-bottomed plates $\left(4 \times 10^{5}\right.$ cells $/ 200 \mu \mathrm{l} /$ well $)$ for $2-5$ days. After removing $100 \mu \mathrm{l}$ of supernatant per well on the day of harvest, $10 \mu \mathrm{l}$ Alamar Blue (Biosource International, Camarillo, California, USA) was added to each well and the cells were cultured for additional 4 hours. The cell number in each well was assessed according to $\mathrm{OD}_{590 \mathrm{~nm}}$ using a CytoFluor apparatus (PerSeptive Biosystems, Framingham, Massachusetts, USA).

Mouse cytokine assays. Cytokines in the culture supernatants of mouse spleen cells were measured with commercial ELISA kits from Endogen (Cambridge, Massachusetts, USA) or R\&D Systems (Minneapolis, Minnesota, USA).

Mouse cytotoxic T lymphocyte (CTL) assay. Transgenic mice carrying $\mathrm{L}^{\mathrm{d}}$-specific TCR (2C mice) were used in this experiment. In the $2 \mathrm{C}$ mice, the majority $(\sim 75 \%)$ of their $\mathrm{T}$ cells are $\mathrm{CD}^{+}$, and almost all the $\mathrm{CD}^{+}$cells carry clonotypic TCR recognized by mAb $1 \mathrm{~B} 2$ (17). The $2 \mathrm{C}$ mice in our colony are of an $\mathrm{H}-2^{\mathrm{b}}$ background (13). 2C spleen cells were stimulated with an equal number of mitomycin C-treated BALB/c spleen cells in 24-well plates at a final density of $4 \times 10^{6}$ cells $/ 0.2$ $\mathrm{ml} /$ well. After 5 days of culture in the presence of 10 $\mathrm{U} / \mathrm{ml}$ recombinant human IL- 2 , and TR6 $(20 \mu \mathrm{g} / \mathrm{ml})$, TR6-Fc $(20 \mu \mathrm{g} / \mathrm{ml})$, or normal human $\operatorname{IgG}(20 \mu \mathrm{g} / \mathrm{ml})$, the viable cells were counted and assayed for their $\mathrm{H}$ $2^{\mathrm{d}}$-specific cytotoxic activity using ${ }^{51} \mathrm{Cr}$-labeled P815 cells $\left(\mathrm{H}-2^{\mathrm{d}}\right)$ as targets. A standard 4-hour ${ }^{51} \mathrm{Cr}$-release assay (13) was carried out in 96-well round-bottomed plates with $0.15 \times 10^{6}$ target cells $/ 200 \mu \mathrm{l} /$ well at different ratios of effector/target cells (10:1, 3:1, 1:1, and $0.3: 1)$. After 4 hours of incubation, $100 \mu \mathrm{l}$ of supernatant was collected from each well and counted in a $\gamma$-counter. The percentage lysis of the test sample is calculated as follows: $\%$ lysis $=(\mathrm{cpm}$ of the test sam- 


\section{Table 1}

$\mathrm{K}_{\text {eq }}$ values for LIGHT binding to TR6-Fc, TR6, and TR2-Fc as determined by BIAcore analysis

\begin{tabular}{lccc}
\hline & TR6 & TR6- $\mathrm{Fc}$ & TR2-Fc \\
$\mathrm{K}_{\text {eq }}($ mean $\pm \mathrm{SD})$ & $10.1 \pm 4.2 \mathrm{nM}$ & $3.87 \pm 1.6 \mathrm{nM}$ & $3.34 \pm 1.7 \mathrm{nM}$ \\
$\chi^{2}$ & 1.82 & 3.57 & 0.46
\end{tabular}

The kinetics of binding between LIGHT and TR6, LIGHT and TR6-Fc, and LIGHT and TR2-Fc were determined by BIAcore sensor chip analysis. The association constant $K_{\mathrm{a}}$, dissociation constant $K_{d}$ and equilibrium constant $\mathrm{K}_{\text {eq }}$ were calculated from the binding sensorgrams using a BIAevaluation software. $K_{e q}$ (mean $\pm \mathrm{SD}$ ) presented was derived from $K_{\mathrm{a}}$ and $K_{\mathrm{d}}$ of two independent experiments. $\chi^{2}$ values were calculated using BIAevaluation software.

ple - cpm of spontaneous release)/(cpm of maximal release - cpm of spontaneous release), where the spontaneous release is derived from $100 \mu$ supernatant of the target cells cultured alone for 4 hours, and the maximal release is derived from a $100 \mu \mathrm{l}$ lysate of $0.15 \times 10^{6}$ target cells that were lysed by SDS in a total volume of $200 \mu \mathrm{l}$.

Mouse heart transplantation. Three- to four-month-old $\mathrm{C} 57 \mathrm{BL} / 6$ mice $\left(\mathrm{H}-2^{\mathrm{b}}\right)$ were used as recipients, and 2- to 3 -month-old BALB/c mice ( $\mathrm{H}-2^{\mathrm{d}}$ ) were used as donors. The procedure of heterotopic heart transplantation has been detailed previously (13). The contraction of the transplanted heart was assessed daily by abdominal palpation. The duration between the day of the operation and the first day when a graft totally lost its palpable activity was defined as the graft survival time. Animals that lost palpable activity of the graft within 3 days after transplantation were classified as technical failures, which was $0 \%$ in this study.

\section{Results}

Preparation of recombinant proteins of buman TR6-Fc, TR6, LIGHT, TR2-Fc, TR11-Fc, and MCIF-Fc. Two forms of TR6 were prepared in this study, a full-length TR6 and a full-length TR6 fused with $\mathrm{Fc}$ (TR6-Fc). $\mathrm{NH}_{2}-$ terminal sequencing revealed that the mature secreted TR6-Fc had the predicted sequence of VAETP starting at amino acid 30 . The estimated purity of the protein preparation was more than $95 \%$, according to SDS-PAGE. Human TR6 without Fc, LIGHT, TR2-Fc, TR11-Fc, and MCIF-Fc were also prepared to a similar purity as TR6-Fc, and their authenticity was verified with $\mathrm{NH}_{2}$-terminal sequencing. Endotoxin levels in the purified proteins were below $10 \mathrm{EU} / \mathrm{mg}$.

The kinetics of binding between TR6 and LIGHT. TR6-Fc has been previously shown to bind both LIGHT and FasL. However, the non-Fc form was not analyzed in those studies. Therefore, we determined the kinetics of binding of LIGHT to both the Fc and non-Fc versions of TR6 using BIAcore analysis. The association constant $K_{\mathrm{a}}$ and dissociation constant $K_{\mathrm{d}}$ were determined and used to calculate the equilibrium constant, $\mathrm{K}_{\text {eq }}$, using the BIAevaluation kinetic software. The $\mathrm{K}_{\mathrm{eq}}$ 's for LIGHT binding to TR6 non-Fc forms, TR6-Fc, and TR2-Fc were $10.1 \pm 4.2 \mathrm{nM}, 3.87 \pm 1.6$ $\mathrm{nM}$, and $3.34 \pm 1.7 \mathrm{nM}$, respectively (Table 1 ). These values had no statistically significant difference according to results of two independent experiments. The $\chi^{2}$ analysis of the $\mathrm{K}_{\mathrm{eq}}$ 's resulted in values below 10 for the bindings between LIGHT/TR6, LIGHT/ TR6-Fc and LIGHT/TR2-Fc (Table 1), indicating that the experimental data fit well to a 1:1 binding model.

TR6-Fc binds LIGHT and competes with TR2 for the binding of LIGHT overexpressed on 293 cell surface. We showed that TR6-Fc could bind to LIGHT on the BIAcore chips. Next we determined whether it could bind to LIGHT expressed on cell surfaces. This was first tested on 293 cells overexpressing LIGHT by flow cytometry. TR6-Fc could bind to the LIGHT-transfectants (Figure 1a, top panel of the middle column, solid line), but not to the untransfected cells (shaded area). The specificity of the binding was further demonstrated by competition of TR6-Fc binding with soluble non-Fc form of TR6 or LIGHT, and nearly complete inhibition was achieved with $10 \mu \mathrm{g}$ of TR6 or LIGHT (second and third panels of the middle column, respectively).

It has been reported that TR2 can bind to LIGHT. Given that TR6 also bound to LIGHT as described earlier here, would it interfere with the binding between TR2 and LIGHT? This possibility was examined with flow cytometry. TR2-Fc could bind to the 293 cells overexpressing LIGHT as expected (Figure 1a, top panel of the left column, solid line), but not to wildtype 293 cells (shaded area). TR6 could competitively displace the binding. At $10 \mu \mathrm{g}$ of TR 6 or LIGHT, the binding of TR2-Fc to the 293 cells was almost completely inhibited (second and third panels of the left column, respectively). TR11-Fc was used as an additional control for possible Fc binding, and it did not bind to the transfected cells (Figure 1a, right column).

The results from this section indicate that TR- 6 can bind to the cell membrane LIGHT, and it can also compete with TR2 for the binding of LIGHT.

TR6 binds LIGHT expressed on activated T cells. LIGHT expression is upregulated on T cells activated with antiCD3 and IL-2 followed by PMA and ionomycin treatment (4). Using this activation regimen, we showed that TR2-Fc bound to the activated T cells (Figure 1b, solid line, top panel of the left column; shaded area represents resting T cells). Soluble TR6 or LIGHT could displace the TR2-Fc binding (second and third panel, left column, respectively), suggesting that the TR2-Fc binding was via LIGHT. We extended this observation by showing that as with TR2-Fc, TR6-Fc also bound to these activated $T$ cells (Figure 1b, top panel of the middle column). Soluble TR6 or LIGHT could displace the TR6-Fc binding, suggesting that the binding was via LIGHT. The control Fc fusion protein TR11-Fc did not bind to these cells (Figure 1b, right column) as expected. These results demonstrate that soluble TR6 can associate with endogenous LIGHT expressed on the activated $T$ cells and that it can interfere with the interaction between LIGHT and TR2 on these cells. This conclusion is valid unless the soluble LIGHT binds to TR6-Fc or TR2-Fc at the same binding site that they 


\section{Figure 1}

TR- 6 binds to cell surface LIGHT and competes with TR2 for its binding to LIGHT. (a) Flow cytometry on LIGHT-expressing 293 cells. LIGHT-expressing 293 transfectants or untransfected 293 cells were incubated with TR2-Fc, TR6-Fc, or a control protein TR11-Fc (50 $\mathrm{ng} /$ sample), and binding was detected with a PE-conjugated goat anti-human Ig antibody by flow cytometry. Solid lines: LIGHTexpressing 293 cells; shaded areas: untransfected 293 cells. Top row: cells were stained in the absence of competitors; middle and bottom rows: cells were stained in the presence of $10 \mu \mathrm{g}$ non-Fc TR6 or LIGHT, respectively, as competitors. (b) Flow cytometry on human T cells. Human T cells were activated as described in Methods. Binding of TR2-Fc, TR6-Fc, or TR11-Fc (50 ng/sample) with T cells was detected by flow cytometry using the PE-conjugated goat antihuman Ig antibody. Solid lines: T cells activated with anti-CD3 followed by ionomycin and PMA; shaded area: nonactivated T cells. Top row: cells were stained in the absence competitors; middle and bottom rows: cells were stained in the presence of $10 \mu \mathrm{g}$ non-FC TR6 or LIGHT, respectively, as competitors.

use to associate with another molecule on the activated $\mathrm{T}$ cells, and thus blocks the binding of TR2-Fc or TR6-Fc to the activated T cells. However, this is only a theoretic speculation without much support of evidence at the present time.

Human TR6-Fc cross-reacts with mouse LIGHT. To investigate the function of TR6 in vivo in mouse models, we needed to establish whether human TR6 could crossreact with mouse LIGHT. For this purpose, we generated recombinant proteins of human LIGHT-FLAG (hLIGHT), mouse LIGHT-FLAG (mLIGHT), and human BlyS-FLAG (BlyS), all of which comprised a FLAG tag. As shown in the top panel of Figure 2, these recombinant proteins from supernatants of expression construct-transfected cells could be detected by antiFLAG $\mathrm{mAb}$, and the dominant bands were corresponding to the expected molecular sizes of the recombinant proteins. Human TR6-Fc precipitated both human and mouse LIGHT-FLAG, but not the control BlyS-FLAG as shown in the middle panel of Figure 2. On the other hand, the receptor of BlyS, BCMA-Fc, could be precipitated BlyS-FLAG, but not by human and mouse LIGHT-FLAG (Figure 2, bottom panel). These results indicate that human TR6 cross-reacts with mouse LIGHT and that the interaction is specific.

TR6-Fc inhibits splenocyte alloactivation in mice. For the in vivo experiments as shown here and later in mouse a

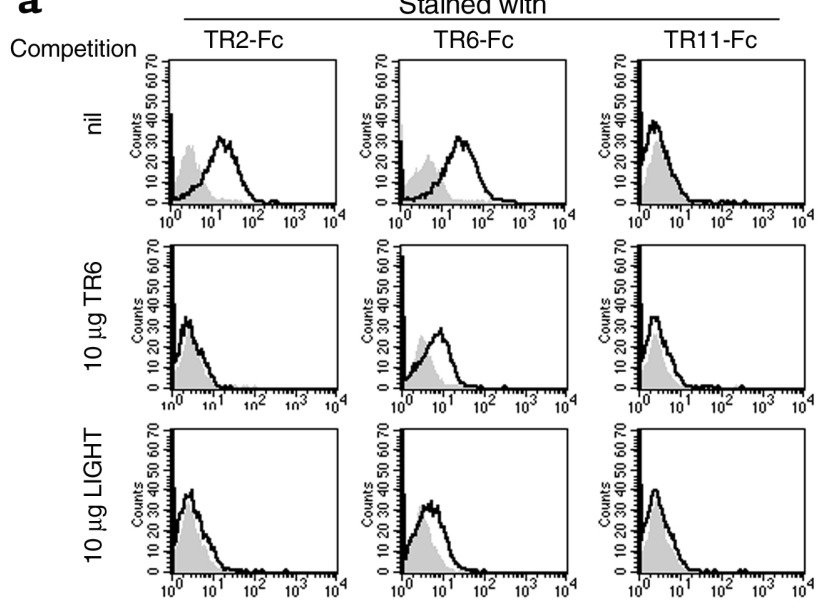

b

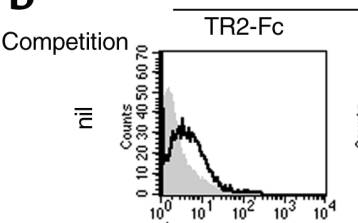

Stained with
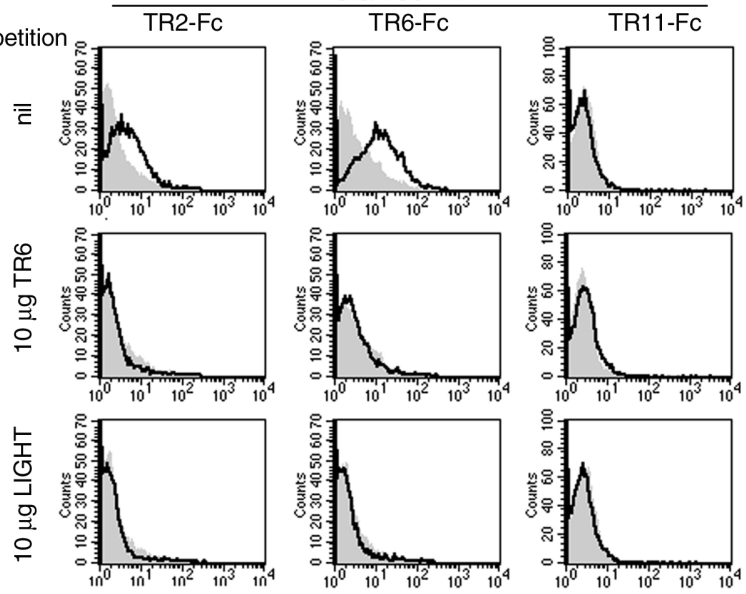

heart transplantation, the TR6-Fc but not TR6 was used because, in general, recombinant protein with an Fc tail decays slower in vivo than one without an Fc tail.

It has been shown previously that $\mathrm{T}$ cells stimulated by alloantigen in vivo have increased spontaneous proliferation ex vivo (18), and alloreactive T cells depend on LIGHT for some costimulation in certain situations (5). We asked whether TR6 had any immune regulatory effects in vivo on alloantigen-stimulated $\mathrm{T}$ cells. Parental splenocytes $\left(\mathrm{H}-2^{\mathrm{b}}\right)$ were transfused intravenously into $\mathrm{H}-2^{\text {bxd }} \mathrm{F} 1$ mice, and this is a typical model

\section{Figure 2}

Human TR6 cross-reacts with mouse LIGHT. Human LIGHT-FLAG, mouse LIGHT-FLAG and human BLyS-FLAG were expressed in HEK 293T cells. Conditioned supernatants were subjected to immunoblot analysis with an anti-FLAG antibody (top panel). A supernatant from vector-transfected cells was included as a control. The supernatants ( $20 \mu \mathrm{l} /$ sample) used represented $5 \%$ of the amounts for immunoprecipitation. The supernatants $(400 \mu \mathrm{l})$ were also subjected to immunoprecipitation either with TR6-Fc (middle panel) or with BCMA-Fc (bottom panel). Immune complexes were resolved by SDS-PAGE and blotted with the anti-FLAG antibody. The prominent bands shown are all of the expected molecular weights of the corresponding recombinant proteins, and the molecular weights are indicated on the left side of the panels. IP, immunoprecipitation.

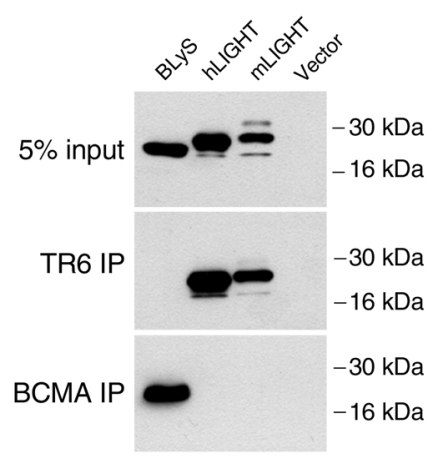



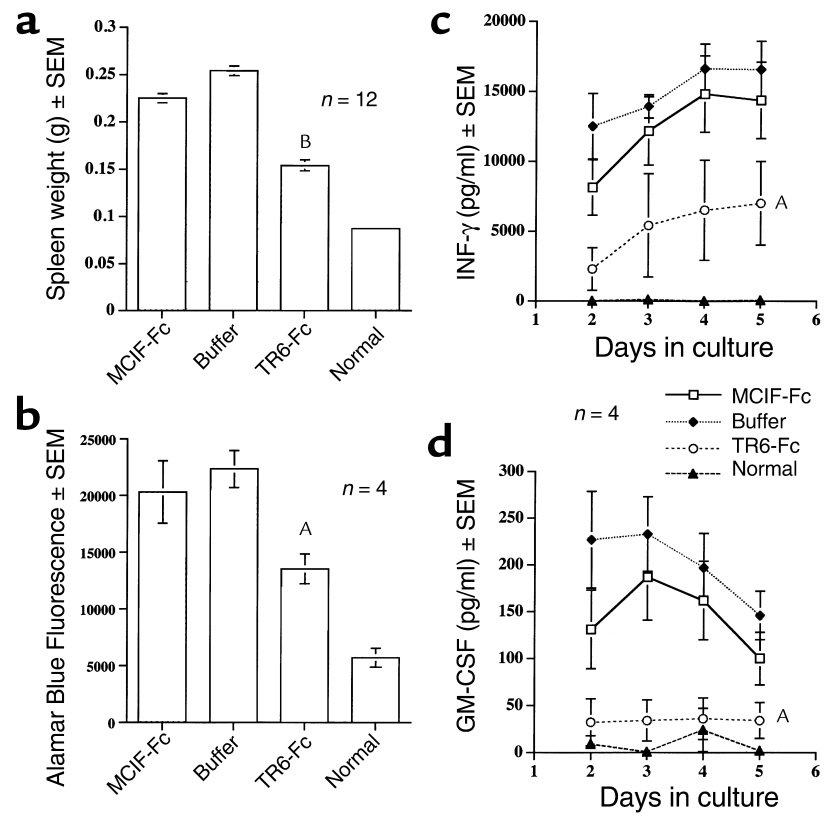

Figure 3

Inhibition of in vivo and ex vivo splenic alloactivation in mice by TR6-Fc. $\mathrm{H}-2^{\text {bxd }} \mathrm{F} 1$ mice were injected intravenously with $1.5 \times 10^{8}$ of $\mathrm{C} 57 \mathrm{BL} / 6\left(\mathrm{H}-2^{\mathrm{b}}\right)$ splenocytes on day 1 and were given daily TR6-Fc, buffer or control protein MCIF-Fc intravenously for 9 days from day -1 to day 8 . Spleen weight (a), spontaneous splenocyte ex vivo proliferation (b), and IFN- $\gamma(\mathbf{c})$ and GM-CSF (d) production were measured. The result of spleen weight is the mean + SEM of three independent experiments ( $n=4$ for each experiment). For splenocyte proliferation, IFN- $\gamma$, and GM-CSF, representative results from one experiment $(n=4)$ are shown, and similar results were obtained in two other experiments. The data were analyzed by ANOVA $t$ tests for spleen weight and splenocyte proliferation. Kinetic cytokine production was analyzed by using mixed effects model for repeated meas-

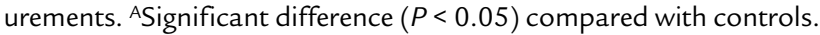
${ }^{B}$ Highly significant difference $(P<0.005)$ compared with controls.

of graft-versus-host response. The recipient mice were given TR6-Fc intravenously at $3 \mathrm{mg} / \mathrm{kg} / \mathrm{d}$ for 9 days starting on day -1 (the day of transfusion was designated as day 1). The F1 mice were sacrificed on day 9, and the spleen weights of the mice were registered. Data from three representative experiments are shown individually in Table 2 , and are also pooled and presented in Figure 3a. Compared with a buffer or control protein (MCIF-Fc) treatment, treatment with TR6-Fc reduced splenomegaly $(P<0.0001)$. The splenocytes were also cultured without additional stimulation to measure their ex vivo proliferation and cytokine production. The proliferation (Figure $3 \mathrm{~b}$ ) as measured on day 4 after the culture, and IFN- $\gamma$ (Figure 3c) and GM-CSF production (Figure $3 \mathrm{~d}$ ) as measured from day 2 to day 5 of the culture, were all suppressed compared with those of a buffer-treated group. No significant changes of these parameters were detected in the group treated with a control protein (MICF$\mathrm{Fc}$ ) compared with those of the buffer-treated

Table 2 F1 mice group. Thus, our results show that TR6-Fc is immunologically active and can indeed modulate $T$ cell-mediated alloactivation (graft-versus-host response) in vivo. In this experiment, however, the inhibited cytokine production, especially in the case of IFN- $\gamma$, could be due to inhibited increase of cell number. The IFN- $\gamma$ level was suppressed about two- to threefold on day 4 , and on this day, the cell number was reduced with a similar degree of twofold according to the Alamar Blue test. On the other hand, the reduced secretion rate of GM-CSF was clearly a contributing factor, in addition to the cell number, as the rate was reduced about fivefold on day 4. It seems that the mechanisms of the reduced IFN- $\gamma$ and GM-CSF secretion differ in this model.

TR6-Fc and TR6 inbibits mouse CTL activity developed against alloantigens. We next used $\mathrm{L}^{\mathrm{d}}$-specific transgenic $2 \mathrm{C} \mathrm{T}$ cells as a model system to evaluate the effect of TR6 on the differentiation of alloantigen-specific CD8 cells into effector cells, given that the high frequency of alloreactive CD8 CTL precursors in the 2C mice enables easy detection of possible changes exerted by TR6. As shown in Figure 4, in the presence of either TR6-Fc or TR6, the CTL activities at the 10:1 and 3:1 effector/target ratios were decreased significantly compared with the cultures containing no recombinant protein or containing normal human $\operatorname{IgG}(P<0.01)$. The detection of a similar effect of TR6 and TR6-Fc in this experiment is of importance, because it excludes the possibility that the effect seen with TR6-Fc is Fc mediated in the mouse system.

TR6-Fc modulates lymphokine production of $2 \mathrm{C} \mathrm{T}$ cells stimulated with $\mathrm{H}-2^{d}$ alloantigens in vitro. CTL differentiation and maturation are modulated by a plethora of lymphokines, and we examined the production of various lymphokines produced by $2 \mathrm{C}$ spleen cells upon stimulation of mitomycin C-treated BALB/c spleen cells $\left(\mathrm{H}-2^{\mathrm{d}}\right)$ in the presence of TR6-Fc (Figure 5). There was a suppression of IL-2 and IL-5 and GM-CSF production between 24 and 72 hours after the stimulation, whereas the IFN- $\gamma$ level was not affected. On the other hand, the levels of IL- 6 and IL-10 were upregulated. We also tested IL-17 and TNF- $\alpha$, but no apparent changes were found. These effects were not caused by direct activation of cytokine-producing cells by

Effect of TR6-Fc on alloactivation-induced splenomegaly in BALB/C x C57BL/6

\begin{tabular}{lcccc}
\hline \multicolumn{5}{c}{ Spleen weight $(\mathrm{g}) \pm$ SEM } \\
Treatment & $\begin{array}{c}\text { Transfer of } \\
\text { C57BL/6 splenocyte }\end{array}$ & $\begin{array}{c}\text { Experiment 1 } \\
(n=4)\end{array}$ & $\begin{array}{c}\text { Experiment 2 } \\
(n=4)\end{array}$ & $\begin{array}{c}\text { Experiment 3 } \\
(n=4)\end{array}$ \\
MCIF-Fc & $1.5 \times 10^{8}$ & $0.233 \pm 0.009$ & $0.223 \pm 0.004$ & $0.219 \pm 0.013$ \\
Buffer & $1.5 \times 10^{8}$ & $0.264 \pm 0.002$ & $0.255 \pm 0.006$ & $0.243 \pm 0.012$ \\
TR6-Fc & $1.5 \times 10^{8}$ & $0.142 \pm 0.017^{\mathrm{A}}$ & $0.149 \pm 0.003^{\mathrm{B}}$ & $0.167 \pm 0.009^{\mathrm{C}}$ \\
Normal & 0 & $0.087 \pm 0.002$ & $0.094 \pm 0.005$ & $0.08 \pm 0.004$
\end{tabular}

The F1 mice were transfused with C57BL/ 6 spleen cells, and administrated with buffer, control protein MCIF-Fc or TR6-Fc intravenously from day -1 to day 8 . The spleens were harvested on day 9 , and their weights were registered. The $P$ value was determined by ANOVA $t$ test with buffer and MCIF-FC groups as controls. ${ }^{A} P<0.0001 ;{ }^{B} P<0.005 ;{ }^{C} P<0.05$ 


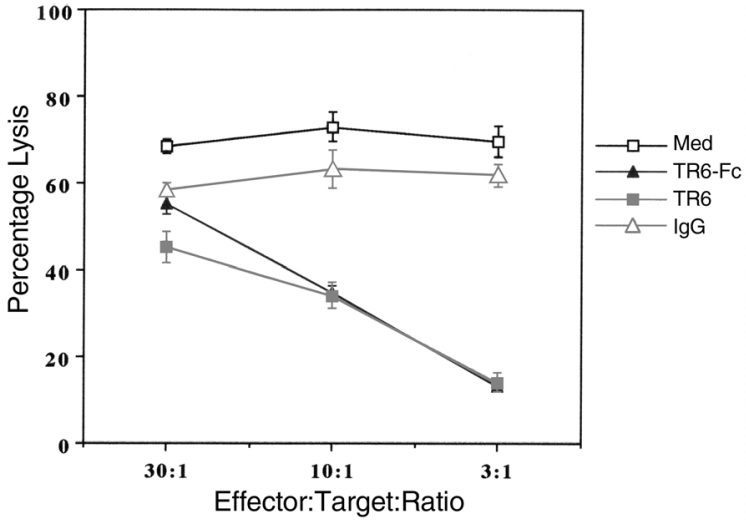

Figure 4

TR6-Fc and TR6 represses the development of CTL in mice. Spleen cells from $2 C$ transgenic mice were stimulated with mitomycin C-treated BALB/c spleen cells, and cultured for 6 days in the presence of TR6-Fc $(20 \mu \mathrm{g} / \mathrm{ml})$, TR6 $(20 \mu \mathrm{g} / \mathrm{ml})$, or normal human IgG $(20 \mu \mathrm{g} / \mathrm{ml})$. The percentage of lysis of the target P815 cells by the effector CTL at different effector/target cell ratios as indicated was measured with a standard $4-\mathrm{h}{ }^{51} \mathrm{Cr}$ release assay with samples in triplicate. At the effector/target ratios of $10: 1$ and 3:1, the CTL activity of the human IgG group or the medium group was significantly higher than that of TR6 or TR6-Fc groups. Data of a representative experiment are shown, and a similar result was obtained from an additional experiment. The CTL activities of TR6- or TR6-Fc-treated versus that of medium- or IgG-treated samples were highly different at 10:1 or $3: 1$ ratio (all $P$ values $<0.01$, ANOVA $t$ test).

TR6-Fc, as TR6-Fc had no effect on cytokine production by resting $2 \mathrm{C}$ spleen cells (data not shown). Although we are yet to form a meaningful interpretation for all the cytokine changes detected in this system, the reduced IL- 2 and increased IL-10 levels probably contributed to the inhibitory effect of TR 6 on the CTL development. In this MLR experiment, the T cells did not progress to the $S$ phase until 72 hours, whereas most of the cytokine levels changed within 72 hours. Thus, the rates of cytokine secretion but not changes of cell number were responsible for the observed modulation of cytokine secretion.

TR6-Fc prolongs heart allograft survival of the mice. Because TR6-Fc could repress graft-versus-host reaction in vivo and inhibit CTL development in in vitro, we speculated that it could also modulate a more complete immune response such as graft rejection. This was tested in a model of mouse heterotopic heart allografting, with $\mathrm{C} 57 \mathrm{BL} / 6$ as recipients and $\mathrm{BALB} / \mathrm{c}$ as donors. The recipients were administrated with TR6-Fc intravenously daily at $7.5 \mathrm{mg} / \mathrm{kg} / \mathrm{d}$ for 7 days starting from one day before the operation. For this test group, the mean survival time (MST) of the grafts was $10.0 \pm 1.2$ days, while the MST of the buffer-treated or control protein MCIF-Fc-treated groups was $6.8 \pm 0.4$ and $6.7 \pm 0.5$ days, respectively (Figure 6 ). The difference between the former and the latter two groups was highly significant $(P=0.0002$, Kaplan-Meier analysis). This result shows that TR6-Fc could modulate an authentic in vivo immune response such as allograft rejection.

\section{Discussion}

In this study, we explored the role of TR6, a new member of the TNFR family, in immune responses. Prominent findings are that TR 6 could compete with TR2 for its binding with LIGHT and that TR6 could downregulate alloresponsiveness of the $\mathrm{T}$ cells.

For full activation of the $\mathrm{T}$ cells, costimulatory signals in addition to TCR cross-linking are required. Costimulation via CD28 on the T cells is a well-established dominant pathway (19-23). However, recent studies suggest that additional costimulatory pathways also exist and that they may have roles at different stages of T-cell activation or on different subsets of $\mathrm{T}$ cells or contribute to the development of different effector functions. For example, signals through inducible costimulator (ICOS) have effects on activated $\mathrm{T}$ cells only, and this pathway enhances IL-10 but not IL-2 production (24); costimulation via signaling lymphocyte activation molecule (SLAM/CD150) enhances IFN- $\gamma$ production and directs memory $\mathrm{T}$ cells toward Th0/Th1 responses (25); crosslink of 4-1BB preferentially promotes CD8 cell proliferation (26); ligation of OX40 increases Th2 responses (27). In the past 2 years, several laboratories have demonstrated that a TNFR family member, TR2, is constitutively
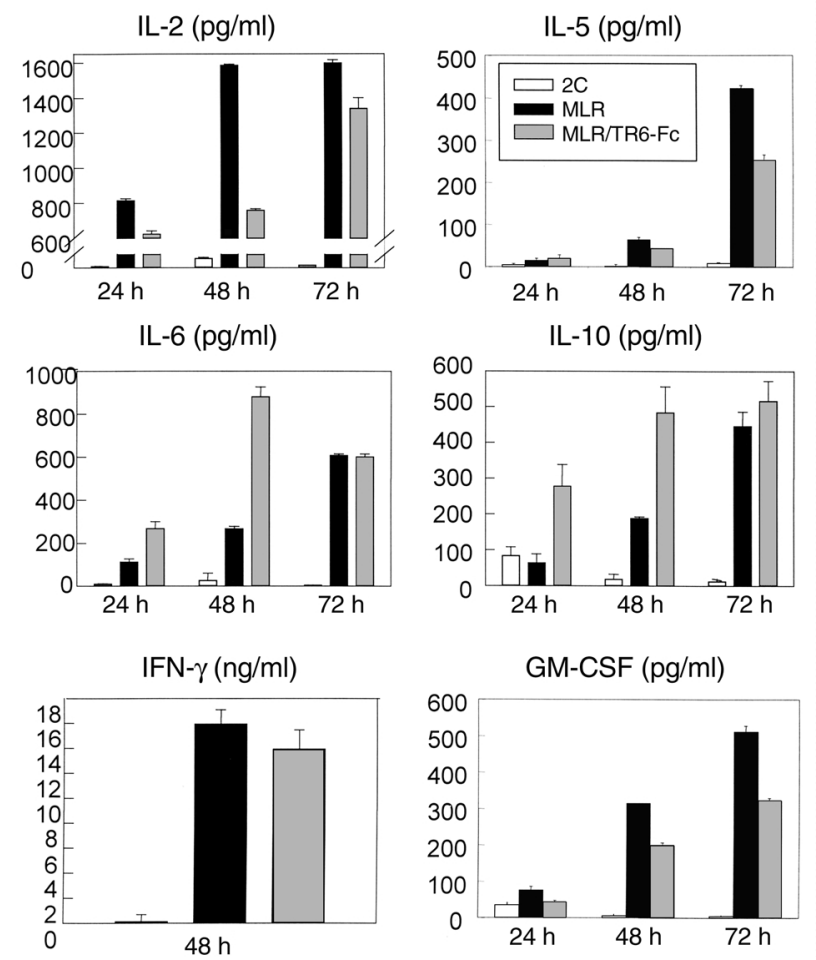

\section{Figure 5}

TR6-Fc modulates in vitro lymphokine production in $\mathrm{H}-2^{\mathrm{d}}$ alloantigen-stimulated $2 \mathrm{C}$ spleen cells. Spleen cells of the $2 \mathrm{C}$ mice were stimulated with mitomycin C-treated BALB/c spleen cells in the absence or presence of TR6-Fc $(20 \mu \mathrm{g} / \mathrm{ml})$. The culture supernatants were collected at 24, 48, and 72 hours for the measurement of lymphokines (IL-2, IL-5, IL-6, IL-10, IFN- $\gamma$, and GM-CSF, as indicated) by ELISA. All the experiments were performed more than twice, and results were consistent. A representative set of data is shown. 


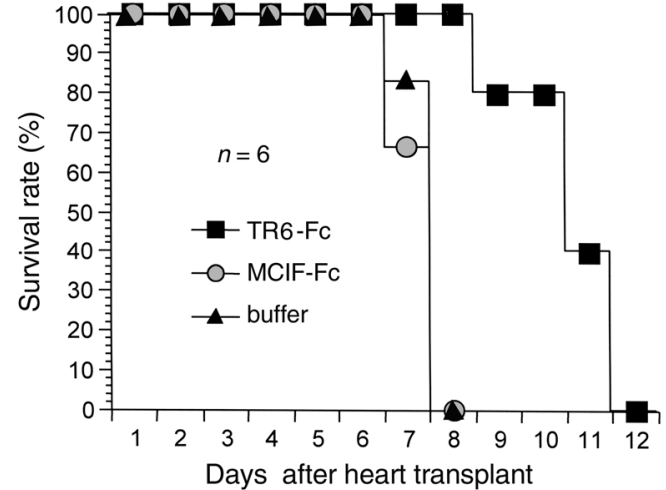

\begin{tabular}{llll}
\hline Group & Survival day & MST+SD & $P$ value \\
Buffer & $6,7,7,7,7,7$ & $6.8 \pm 0.4$ & -- \\
MCIF-Fc & $6,6,7,7,7,7$ & $6.7 \pm 0.5$ & 0.55 \\
TR6-Fc $^{\text {A }}$ & $8,10,10,11,11$ & $10 \pm 1.2$ & 0.0002 \\
\hline
\end{tabular}

TR6-Fc i.v. $7.5 \mathrm{mg} / \mathrm{kg} / \mathrm{d}$

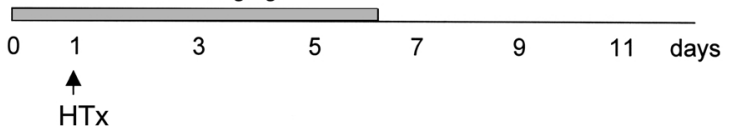

\begin{abstract}
Figure 6
TR6-Fc prolongs heart allograft survival of the mice. C57BL/6 mice were transplanted heterotopically with BALB/c hearts. TR6-Fc, MCIF-Fc (7.5 $\mathrm{mg} / \mathrm{kg}$ ), or buffer was given intravenously daily for 7 days, starting 1 day before the operation. The survival rates of the heart allografts are depicted in the graph. The survival days of each graft, MST (mean survival time), and $P$ value (Kaplan-Meier analysis) between the buffertreated and protein groups are shown in the inset table. The regimen of TR6-Fc administration is illustrated in the bottom. HTx, heart transplantation; i.v., intravenous. AHighly significant statistically.
\end{abstract}

expressed on $\mathrm{T}$ cells and can transduce costimulatory signals into $T$ cells $(10,12)$. It is also demonstrated that LIGHT is the ligand of TR2 (4) and provides costimulation to the TR2-expressing cells.

The function of TR6 in an immune response, other than being a decoy receptor interfering with Fas$\mathrm{L}$-induced apoptosis, is not understood. Given that TR6 can also bind LIGHT, there is a possibility that it might interfere with costimulation via the LIGHT-TR2 interaction. In this study, we first produced recombinant TR6 and showed that it was biologically active in terms of binding to either recombinant LIGHT on BIAcore chips, to 293 cells overexpressing LIGHT, or to endogenous LIGHT expressed on activated T cells. We then demonstrated, for the first time to our knowledge, that the soluble TR6 could indeed compete with TR2 for its binding of LIGHT expressed on the cell surface. Subsequent experiments showed that the soluble TR6 could downregulate MLR and suppress alloantigen-stimulated IFN- $\gamma$ and GM-CSF production ex vivo, inhibit CTL development in vitro, and ameliorate heart allograft rejection in vivo. One of the plausible mechanisms for these observed immunomodulatory effects of TR 6 is its interference with the LIGHT-TR2 costimulation pathway.
We noticed that inhibition of proliferation in the mouse in vivo MLR (the graft-versus-host response) by TR6 was not complete even with high concentrations of TR6. The same was also true for the effect of TR 6 on IL-2 production in in vitro MLR and on graft rejection in vivo. Further increase of the TR6-Fc concentration did not augment the degree of inhibition. This is probably due to the fact that costimulation via TR2 is only a part of the costimulation program for the $\mathrm{T}$ cells. Considering that CD28 plays a dominant role in costimulation, and that multiple costimulation pathways coexist as discussed earlier here, the partial inhibition is within our expectation. However, we still cannot exclude the possibility that the partial inhibition is due to selective effects of TR6 on a subpopulation of T cells.

Although we speculate that the interference of the stimulation from LIGHT to TR2 is one of the possible mechanisms of the effect of TR6 observed, other possibilities are worth discussing. During T-cell activation, FasL is upregulated in T cells and FasL is responsible for activation-induced T-cell death. TR6 can bind to FasL and inhibit FasL-induced apoptosis in some experimental models (1). However, such a mechanism will only enhance in theory the viability of the $\mathrm{T}$ cells, but will not downregulate the proliferation of them. In the in vitro CTL assay, TR6-Fc or TR6 was only present during the priming stage, during which the cytotoxic $T$ cells differentiated, but not in the 4-hour assay period, during which the killing took place. The repressed the CTL activity thus had nothing to do with the possible interference of TR6 with the Fas/ FasL interaction for the killing, but appears to be due to a regulatory effect of TR6 during CTL differentiation. Indeed, if TR6-Fc was only presented during the 4hour CTL assay period, no effect was noticed (data not shown). Allograft rejection is not dependent on the Fas-FasL interaction, as the rejection response in Fas mutant B6.MRL-lpr mice is not impaired $(28,29)$, whereas the graft rejection in the TR6-Fc-treated mice was ameliorated in our study. These results as a whole suggest that the observed effects of TR6-Fc on the several in vitro and in vivo immune responses as examined in our study are not mediated via the Fas-FasL pathway due to their role in apoptosis induction. However, we cannot rule out the possibility that TR6 might interfere with costimulation conducted via unior bidirectional interaction between Fas and FasL during the induction phase of the immune response. TR6 might also interfere with possible reverse signaling from TR6 to LIGHT.

In addition to TR2, LIGHT also binds LTßR (4). Such binding is necessary and sufficient to induce apoptosis of LT $\beta$ R-expressing cells (7). However, LT $\beta R$ is not expressed on T cells (30), and logically the downregulation of the MLR and cytokine production cannot be attributed to reduced apoptosis mediated via LT $\beta R$. LT $\beta R$ is essential in lymphoid organogenesis and germinal center formation that is required in T-dependent antibody production $(31,32)$. That LT $\beta$ knockout mice 
can develop such defects suggests that LIGHT interaction with LT $\beta$ R does not suffice to compensate the role of LT $\beta$. Consequently, the possible interference of TR6 with the LIGHT and LT $\beta$ R interaction will unlikely affect the humoral immune response. Besides, in the in vitro and in vivo MLR, and in the first 10 days of graft rejection as tested in our study, the humoral response has no essential role.

What is the function of endogenous TR6 in the immune system? TR6 is a secreted protein according to its sequence characteristics. Moreover, when TR6 was expressed in CHO cells, 239 cells, and sf9 cells, it secreted into the supernatants, from which we purified the recombinant TR6 or TR6-Fc (the data of the first two cell types not shown). In the human system, TR6 protein can indeed be detected in T-cell culture supernatants, and its level is increased after mitogen stimulation (data not shown). It is conceivable that the increased level of soluble TR6 will enhance its regulatory effect once an immune response is ongoing. According to our current knowledge including that reported here, TR6 will likely block the interaction between certain pairs of TNF and TNFR members, LIGHT/TR2 and Fas/FasL being two of them. We cannot exclude the possibility that TR6 might also interfere with the interaction between other known or unknown TNF and TNFR pairs. The end result of the TR6's activity might depend on the TNF/TNFR pairs with which it interferes. The complex way of cytokine modulation by the recombinant TR6, which suppressed IL-2, IL-5, and GM-CSF production, enhanced IL-6 and IL-10 levels, and had no significant effects on IFN- $\gamma$ secretion in MLR in vitro, is difficult to be explained simply by its interference on the one-way costimulation from LIGHT to TR2; rather it might just be a reflection of TR6's effects on different TNF/TNFR pairs and/or on different subsets of cells expressing these molecules. We are currently exploring such additional immunoregulatory mechanisms of TR6. It is to be noted that certain tumors can also produce TR6, and this might be a mechanism for tumors to interfere the immune surveillance.

The use of $\mathrm{Fc}$ to make recombinant protein has the advantage of generating stable proteins with a detectable tag. On the other hand, a potential pitfall exists in that the $\mathrm{Fc}$ fusion protein could kill cells by activating the complement and/or by antibodydependent cytotoxicity (ADCC). IL-2R-Fc has been shown to kill IL-2R-positive cells and blocks diabetogenic autoimmunity through these two mechanisms (33). Fc could also exert direct suppressive signals in FcR-positive cells under certain conditions. These potential effects of $\mathrm{Fc}$ are largely irrelevant in our mouse models. The Fc in the fusion TR6 is of human $\mathrm{IgG}_{1}$ and thus binds poorly to mouse $\mathrm{Fc \gamma R}$. This argues against possible roles of ADCC and negative signaling via $F c \gamma R$ in our mouse in vivo MLR (graftversus-host response) and in vivo graft rejection. More convincingly, TR6-Fc and TR6 without the Fc tag had similar inhibitory effects on the development of cytotoxic T-cell activity using $\mathrm{L}^{\mathrm{d}}$-specific mouse $\mathrm{T}$ cells stimulated with $\mathrm{H}-2^{\mathrm{d}}$ cells. Taken together, these data indicate that the immunoregulatory effects of TR6-Fc in our mouse model are independent of the human $\mathrm{Fc}$ tag in the fusion protein.

In conclusion, our study indicates that both in vitro and in vivo TR6 can exert important immunoregulatory functions, some of which have potential for therapeutic applications.

\section{Acknowledgments}

This work was supported in part by grants from the Canadian Institutes of Health Research (MT-15673), Canadian institute of Health Research/Canadian Blood Service Partnership, the Kidney Foundation of Canada, the Heart and Stroke Foundation of Quebec, the National Cancer Institute Breast Cancer Initiative, and the Roche Organ Transplantation Research Foundation (ROTRF \#474950960) to J. Wu, and by the Nephrology Service of the Notre-Dame Hospital and the J-Louis Levesque Foundation. J. Wu is a senior scholar of Quebec Health Research Foundation. We thank Susie Kirchhof and Jeff Carrell for their technical assistance.

1. Pitti, R.M., et al. 1998. Genomic amplification of a decoy receptor for Fas ligand in lung and colon cancer. Nature. 396:699-703.

2. Bai, C., et al. 2000. Overexpression of M68/DcR3 in human gastrointestinal tract tumors independent of gene amplification and its location in a four-gene cluster. Proc. Natl. Acad. Sci. USA. 97:1230-1235.

3. Yu, K.Y., et al. 1999. A newly identified member of tumor necrosis factor receptor superfamily (TR6) suppresses LIGHT-mediated apoptosis. J. Biol. Chem. 274:13733-13736.

4. Mauri, D.N., et al. 1998. LIGHT, a new member of the TNF superfamily, and lymphotoxin alpha are ligands for herpesvirus entry mediator. Immunity. 8:21-30.

5. Tamada, K., et al. 2000. LIGHT, a TNF-like molecule, costimulates T cell proliferation and is required for dendritic cell-mediated allogeneic $\mathrm{T}$ cell response. J. Immunol. 164:4105-4110.

6. Zhai, Y., et al. 1998. LIGHT, a novel ligand for lymphotoxin beta receptor and TR2/HVEM induces apoptosis and suppresses in vivo tumor formation via gene transfer. J. Clin. Invest. 102:1142-1151.

7. Rooney, I.A., et al. 2000. The lymphotoxin-beta receptor is necessary and sufficient for LIGHT-mediated apoptosis of tumor cells. J. Biol. Chem. 275:14307-14315.

8. Browning, J.L., et al. 1997. Characterization of lymphotoxin-alpha beta complexes on the surface of mouse lymphocytes. J. Immunol. 159:3288-3298.

9. Harrop, J.A., et al. 1998. Antibodies to TR2 (herpesvirus entry mediator), a new member of the TNF receptor superfamily, block T cell proliferation, expression of activation markers, and production of cytokines. $J$. Immunol. 161:1786-1794.

10. Kwon, B.S., et al. 1997. A newly identified member of the tumor necrosis factor receptor superfamily with a wide tissue distribution and involvement in lymphocyte activation. J. Biol. Chem. 272:14272-14276.

11. Harrop, J.A., et al. 1998. Herpesvirus entry mediator ligand (HVEM-L), a novel ligand for HVEM/TR2, stimulates proliferation of T cells and inhibits HT29 cell growth. J. Biol. Chem. 273:27548-27556.

12. Tamada, K., et al. 2000. Modulation of T-cell-mediated immunity in tumor and graft-versus-host disease models through the LIGHT costimulatory pathway. Nat. Med. 6:283-289.

13. Chen, H., et al. 1996. Impaired signaling in alloantigen-specific CD8+ T cells tolerized in vivo: employing a model of $\mathrm{L}^{\mathrm{d}}$-specific TCR transgenic mice transplanted with allogenic hearts under the cover of a short-term rapamycin treatment. J. Immunol. 157:4297-4308.

14. Moore, P.A., et al. 1999. BLyS: member of the tumor necrosis factor family and B lymphocyte stimulator. Science. 285:260-263.

15. Morris, A.E., et al. 1999. Incorporation of an isoleucine zipper motif enhances the biological activity of soluble CD40L (CD154). J. Biol. Chem. 274:418-423.

16. Gross, J.A., et al. 2000. TACI and BCMA are receptors for a TNF homologue implicated in B-cell autoimmune disease. Nature. 404:995-999. 
17. Sha, W.C., et al. 1988. Selective expression of an antigen receptor on CD8-bearing T lymphocytes in transgenic mice. Nature. 335:271-274.

18. Via, C.S. 1991. Kinetics of T cell activation in acute and chronic forms of murine graft-versus-host disease. J. Immunol. 146:2603-2609.

19. Schwartz, R.H. 1992. Costimulation of T lymphocytes: the role of CD28, CTLA-4, and B7/BB1 in interleukin-2 production and immunotherapy. Cell. 71:1065-1068.

20. Linsley, P.S., and Ledbetter, J.A. 1993. The role of the CD28 receptor during T cell responses to antigen. Annu. Rev. Immunol. 11:191-212.

21. Allison, J.P. 1994. CD28-B7 interactions in T-cell activation. Curr. Opin. Immunol. 6:414-419.

22. June, C.H., Bluestone, J.A., Nadler, L.M., and Thompson, C.B. 1994. The B7 and CD28 receptor families. Immunol. Today. 15:321-331.

23. Jenkins, M.K. 1994. The ups and downs of T cell costimulation. Immunity. 1:443-446.

24. Hutloff, A., et al. 1999. ICOS is an inducible T-cell co-stimulator structurally and functionally related to CD28. Nature. 397:263-266.

25. Aversa, G., Chang, C.C., Carballido, J.M., Cocks, B.G., and de Vries, J.E. 1997. Engagement of the signaling lymphocytic activation molecule (SLAM) on activated T cells results in IL-2-independent, cyclosporin Asensitive $\mathrm{T}$ cell proliferation and IFN-gamma production. J. Immunol. 158:4036-4044.

26. Shuford, W.W., et al. 1997. 4-1BB costimulatory signals preferentially induce CD8+ $\mathrm{T}$ cell proliferation and lead to the amplification in vivo of cytotoxic T cell responses. J. Exp. Med. 186:47-55.

27. Flynn, S., Toellner, K.M., Raykundalia, C., Goodall, M., and Lane, P. 1988. CD4 $\mathrm{T}$ cell cytokine differentiation: the B cell activation molecule, OX40 ligand, instructs CD4 T cells to express interleukin 4 and upregulates expression of the chemokine receptor, Blr-1. J. Exp. Med. 188:297-304.

28. Li, X.C., et al. 1999. Induction of allograft tolerance in the absence of Fasmediated apoptosis. J. Immunol. 163:2500-2502.

29. Djamali, A., and Odorico, J.S. 1998. Fas-mediated cytotoxicity is not required for rejection of murine nonvascularized heterotopic cardiac allografts. Transplantation. 66:1793-1801.

30. Ware, C.F., VanArsdale, T.L., Crowe, P.D., and Browning, J.L. 1995. The ligands and receptors of the lymphotoxin system. Curr. Top. Microbiol. Immunol. 198:175-218.

31. Alimzhanov, M.B., et al. 1997. Abnormal development of secondary lymphoid tissues in lymphotoxin beta-deficient mice. Proc. Natl. Acad. Sci. USA. 94:9302-9307.

32. Koni, P.A., et al. 1997. Distinct roles in lymphoid organogenesis for lymphotoxins alpha and beta revealed in lymphotoxin beta-deficient mice. Immunity. 6:491-500.

33. Zheng, X.X., et al. 1999. IL-2 receptor-targeted cytolytic IL-2/Fc fusion protein treatment blocks diabetogenic autoimmunity in nonobese diabetic mice. J. Immunol. 163:4041-4048. 